\title{
Don't Try to Control Everything!: An Emergent Morphology Control of a Modular Robot
}

\author{
Akio Ishiguro, Masahiro Shimizu \\ Dept. of Computational Science and Engineering \\ Nagoya University \\ Nagoya, Japan \\ Email: \{ishiguro/shimizu\}@cmplx.cse.nagoya-u.ac.jp
}

\author{
Toshihiro Kawakatsu \\ Dept. of Physics \\ Tohoku University \\ Sendai, Japan \\ Email: kawakatu@cmpt.phys.tohoku.ac.jp
}

\begin{abstract}
This paper discusses a fully decentralized algorithm able to control the morphology of a modular robot, consisting of many identical modules, according to the environment encountered. One of the significant features of our approach is that we explicitly exploit "emergent phenomena" stemming from the interaction between control and mechanical dynamics in order to control the morphology in real time. To this end, we particularly focus on a "functional material" and a "mutual entrainment", the former of which is used as a connection mechanism between the modules, and the latter of which plays as the core of the control mechanism for the generation of locomotion. Simulation results indicate that the proposed algorithm can induce "protoplasmic streaming", which allows us to successfully control the morphology of modular robot in real time according to the situation without losing the coherence of the entire system.
\end{abstract}

\section{INTRODUCTION}

Recently, a modular robot (or called reconfigurable robot), consisting of many mechanical units (hereinafter called modules), have been attracting lots of concern. Since the relative positional relationship among the modules can be altered actively according to the situation encountered, a modular robot is expected to show significant abilities, e.g., adaptability, fault tolerance, scalability, and flexibility, compared with a robot on a fixed-morphology basis [1-5]. Under these circumstances, so far various morphology control methods have been proposed for modular robots. Most of these studies, however, have the following problems:

- Morphological alteration is discussed in some studies, but is usually resolved by turning into a module rearrangement problem in a centralized-planning manner.

- Modules are normally connected mechanically and/or electromagnetically by highly rigid mechanisms.

In order to fully exploit the advantages mentioned above, (1)each module should be controlled in a fully decentralized manner, and (2)the resultant morphology of the entire system should be emerged through the module-to-module and module-to-environment interactions.

In light of these facts, this study is intended to deal with an emergent control method which enables a modular robot to change its morphology in real time according to the situation encountered without the use of any global information as well as without losing the coherence of the entire system. Since there still remains much to be understood about how such emergent systems can be created, in this study, we employ the following working hypothesis: well-balanced coupling between control and mechanical dynamics plays an essential role to elicit interesting emergent phenomena, which can be exploited to increase adaptability, scalability, and so on.

Based on this working hypothesis, here we particularly focus on the exploitation of a functional material and a mutual entrainment among nonlinear oscillators, the former of which is used as a connection mechanism between the modules, and the latter of which plays as the core of the control mechanism for the generation of locomotion. In what follows, we will explain these in more detail. As mentioned before, most modular robots developed so far have their modules connected mechanically and/or electromagnetically by highly rigid mechanisms. Under this kind of connection mechanism, however, the control algorithm required usually ends up to be extremely complicated and intractable since it has to always specify which modules should be connected physically as well as how each module should be moved. In addition, module connections done by such a highly rigid mechanism may impair some of the advantages expected, particularly the flexibility against environmental changes. In order to alleviate this problem, we focused on a functional material. More specifically, we used Velcro strap as a practical example, since this intrinsically have an interesting properties: when the male and female halves of Velcro contact each other, they are connected easily; and when the halves are disconnected by a force greater than the yield strength, they come apart automatically. Exploiting the property of this material itself as a part of the mechanical dynamics is expected not only to reduce the computational cost required for the connection control dramatically, but also to induce emergent properties in morphology control ${ }^{1}$.

For efficient morphology control of a modular robot with this kind of material, the induction of protoplasmic streaming is considered in this study. Protoplasmic streaming is a many-body behavioral phenomenon widely observed

${ }^{1}$ Due to the automatic disconnection by a force beyond the yield strength, this material is expected to absorb the conflict between a modular robot and its environment. When a highly rigid connection mechanism is employed, one has to always control precisely, which will lead to the huge computational cost. Therefore, a functional material can be viewed as a mechanism which autonomously controls the connection and disconnection among the modules by exploiting its intrinsic properties. 
in nature. We expect that this contributes to control the morphology of an entire module group in an emergent manner from the interactions between the modules and between the module group and its surrounding environment. Here, a mutual entrainment plays an essential role to elicit protoplasmic streaming, which will be discussed later.

Since the study is still in the initial stage, this paper deals with the morphology control of a modular robot placed two dimensionally. More specifically, we attempt to construct a control method able to induce protoplasmic streaming inside the modular robot. Simulation results indicate that the proposed method is highly promising.

\section{Proposed Method}

\section{A. The Mechanical Structure}

A modular robot considered in this study consists of many identical modules, each of which has a mechanical structure like the one shown in Fig. 1. Each module is equipped with telescopic arms and a ground friction control mechanism (explained later). Each module is also equipped with two types of light-detecting sensor: one is for detecting the goal; and the other is for ambient light. Note that the module is covered with Velcro straps with different polarities, i.e., male and female halves of Velcro. The dynamics of the connection mechanism is specified by the yield stress of Velcro employed: connection between the modules is established spontaneously where the arms of each module make contact; disconnection occurs if the disconnection stress exceeds the yield stress. We also assume that local communication between the connected modules is possible, which will be used to create phase gradient inside the modular robot (discussed below). In this study, each module is moved by the telescopic actions of the arms and by ground friction. Therefore, each module itself does not have any mobility but can move only by the collaboration with other modules.

\section{B. The Control Algorithm}

Under the above mechanical structure, we consider how we can generate stable and continuous protoplasmic streaming inside the modular robot. As observed in slime mold and other organisms, the generation of an appropriate phase gradient inside the modular robot is indispensable in order to induce protoplasmic streaming. To this end, a nonlinear oscillator is implemented onto each module with

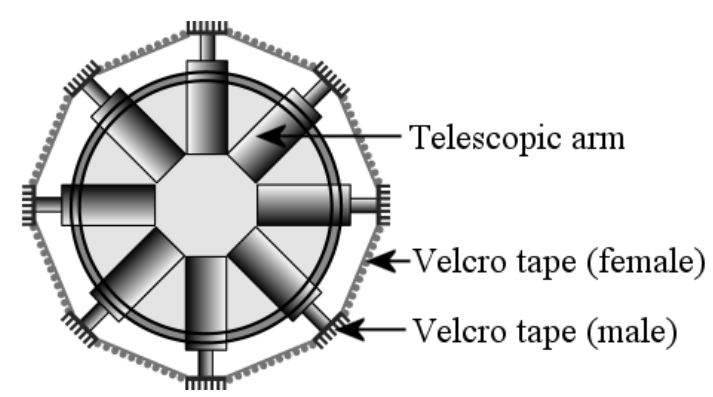

Fig. 1. Mechanical structure of the modular robot employed (top view).

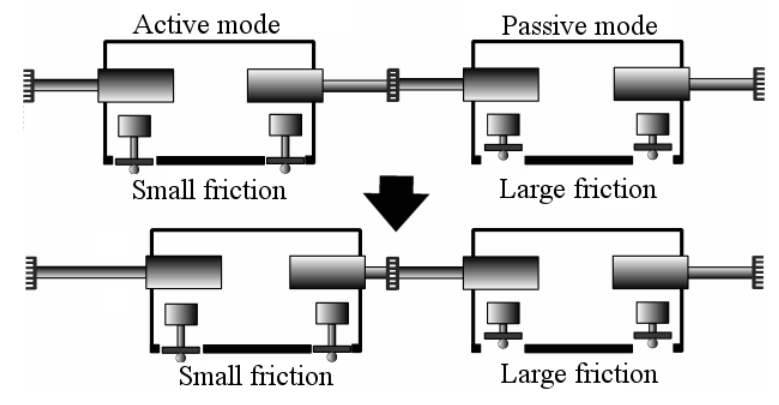

Fig. 2. A schemtic of the active mode and the passive mode (A side view of the connected modules is shown for clarity).

which we expect to create an appropriate equiphase surface suitable for generating protoplasmic streaming through the mutual entrainment among the oscillators. In what follows, we will give a detailed explanation of this algorithm.

1) Active mode and passive mode: Here, the basic operation of each module is defined. Each module in the modular robot can take one of two exclusive modes at any time: active mode and passive mode. As shown in Fig. 2, a module in the active mode actively contracts the connected arms, and simultaneously reduces the ground friction. In contrast, a module in the passive mode increases the ground friction $^{2}$, and return its arms to their original length. Note that a module in the passive mode does not move itself but serves as a supporting point for efficient movement of the module group in the active mode.

2) Configuration of the phase gradient through mutual entrainment: As mentioned before, the modes of each module should be switched appropriately in order to induce a protoplasmic streaming phenomenon. Therefore, the configuration of an equiphase surface is extremely important as a guideline for the mode switching. In this study, the creation of an equiphase surface effective for generating the protoplasmic streaming phenomenon is attempted only through the local communication. To do so, we focused on a mutual entrainment phenomenon created through the interaction among nonlinear oscillators. In the following, we will explain this in more detail.

As a model of a nonlinear oscillator, van der Pol oscillator (hereinafter VDP oscillator) was employed, since this oscillator model is widely used for its significant entrainment property. The equation of VDP oscillator implemented on module $i$ is given by

$$
\alpha_{i} \ddot{x}_{i}-\beta_{i}\left(1-x_{i}^{2}\right) \dot{x}_{i}+x_{i}=0,
$$

where the parameter $\alpha_{i}$ specifies the frequency of the oscillation. $\beta_{i}$ corresponds to the convergence rate to the limit cycle.

The local communication among the physically connected modules is done by the local interaction among the VDP oscillators of these modules. This is conducted by referencing the study of Kakazu et al. [6], which is

\footnotetext{
${ }^{2}$ Slime molds do really use this mechanism. This is called a pseudopod.
} 
expressed as $^{3}$ :

$$
x_{i}(t+1)=x_{i}(t)+\varepsilon\left(\frac{1}{N_{i}(t)} \sum_{j=1}^{N_{i}(t)} x_{j}(t)-x_{i}(t)\right),
$$

where $N_{i}(t)$ represents the number of modules neighboring module $i$ at time $t$. The parameter $\varepsilon$ specifies the strength of the interaction.

When VDP oscillators interact according to Equation (2), significant phase distribution can be created effectively by varying the value of $\alpha_{i}$ in Equation (1) for some of the oscillators [6]. In order to create an equiphase surface effective for the generation of protoplasmic streaming, we set the value of $\alpha_{i}$ as:

$$
\alpha_{i}= \begin{cases}0.7 & \text { (if the goal light is detected) } \\ 1.3 & \text { (if the ambient light is detected) (3) } \\ 1.0 & \text { (otherwise) }\end{cases}
$$

Note that except the modules detecting the goal light, the modules on the boundary, i.e., the outer surface, have the value of $\alpha_{i}=1.3$. This allows us to introduce the effect of surface tension, which is indispensable to maintain the coherence of the entire system. Figure 3 shows the phase distribution when the modules are arranged circularly. The top and bottom of the figure corresponds to the front and rear of the modular robot, respectively. In the figure, arrows - each of which represents the direction of gradient vector at the corresponding point - are also depicted for clarity.

3) Generation of the protoplasmic streaming: Here, we consider a control algorithm able to generate protoplasmic streaming exploiting the phase distribution created from the aforementioned mutual entrainment among the VDP oscillators. To do so, the two possible modes, i.e., the active and passive modes, of each module should be appropriately altered corresponding to the emerged phase distribution. In this study, therefore, we first divide one period $T$, i.e., the period of $(n-1) \pi \leq \theta_{i}(t)<(n+1) \pi$, of the VDP oscillator equally into $N_{p}$ sections. Then, in each phase section corresponding to time $T / N_{p}$ the two mode is altered according to the duty ratio $\gamma$ expressed as:

$$
\gamma=\frac{T_{a}}{T_{a}+T_{p}}
$$

where $T_{a}$ and $T_{p}$ are the period of the active mode and passive mode in time $T / N_{p}$, respectively. Fig. 4 illustrates how the active and passive modes are altered according to the phase of the oscillation. The vertical and horizontal axes are the phase of the VDP oscillator (hereinafter denoted by $\theta_{i}$ ) and the time step, respectively. For clarity, $N_{p}$ is set to two in the figure.

When the duty ratio is set as above under the phase distribution shown in Fig. 3, the timings of the mode

\footnotetext{
${ }^{3}$ In this study, the mutual entrainment among the VDP oscillators adopted by Kakazu et al. [6] is employed to create an appropriate phase gradient. The point of this study, however, is to induce the protoplasmic streaming inside the modular robot by exploiting the interaction between the dynamics of the functional material and the distribution of the velocity vectors created from the resultant shape of the equiphase surface. This is totally different from the study of Kakazu et al., which is aimed at controlling a swarm of motile elements, i.e., autonomous mobile robots.
}

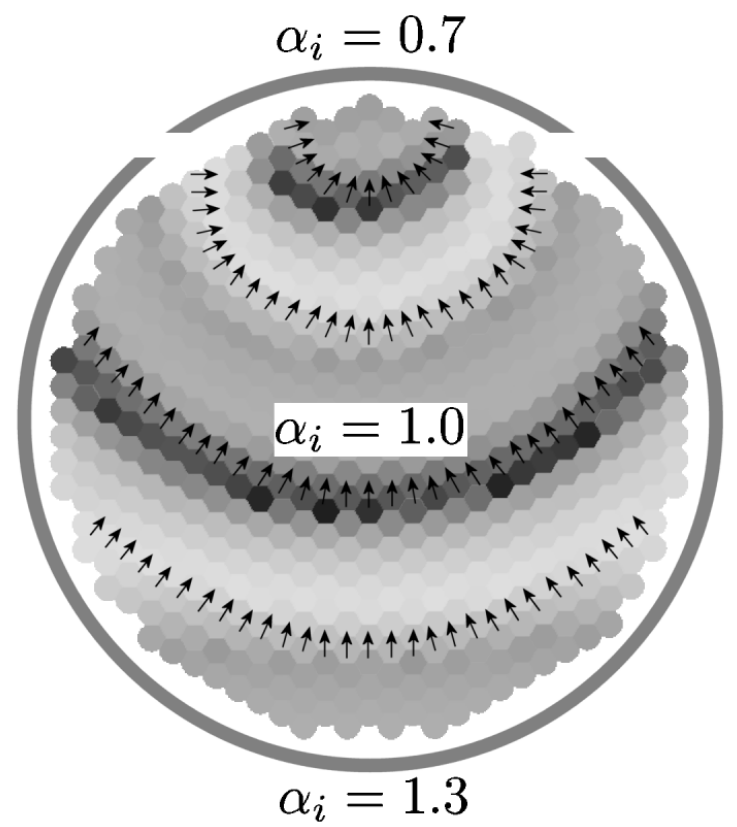

Fig. 3. Phase distribution created through the mutual entrainment among the VDP oscillators in a circular arrangement. The gray scale denotes the value of the phase at the corresponding point.

alternation are propagated from the front to the rear inside the modular robot as traveling waves. In this study, the extension/contraction of each arm of module $i$ in the active mode is determined according to the phase difference with the neighboring module. This is given by

$$
F_{i}^{m}(t)=-k\left\{\theta_{j}(t)-\theta_{i}(t)\right\},
$$

where, $F_{i}^{m}(t)$ is the force applied for the extension/contraction of the $m$-th arm of module $i$ at time $t$. $k$ is the coefficient. $\theta_{j}(t)$ represents the phase of the neighboring module physically connected to module $i$. Due to this, the degree of arm extension/contraction of each module will become most significant along the phase gradient (see Fig. 3).

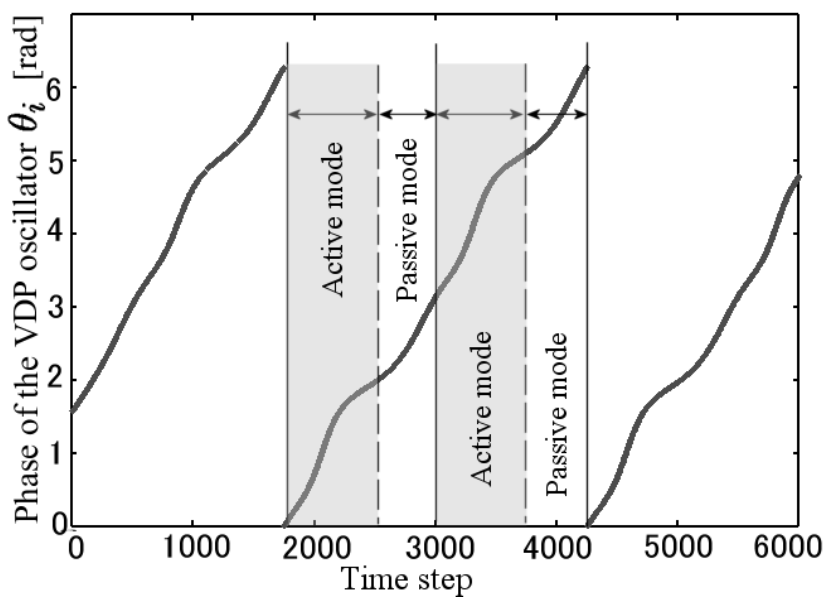

Fig. 4. Mode alternation. 
What should be noticed here can be summarized as: (1) the motion-direction vectors of the modules along the midline connecting from the front to the rear are oriented almost in the same direction; (2) the others are heading inward, the latter of which induces the effect of surface tension (see the arrows in Fig. 3). This enables the entire system to advance forward while maintaining its coherency.

\section{Simulation Results}

\section{A. Problem Setting}

In this study, a phototaxis behavior is adopted as a practical example: the task of the modular robot is to move toward the goal without losing the coherece of the entire system. In the simulation discussed below, the light from the goal is given from the top of the figure, and thus the modular robot moves upward. The simulation conditions employed are as follows:

Initial arrangement: Circular (each module is placed so as to be the most densely filled structure, as shown in Fig. 3).

Parameters of the VDP oscillator: $\beta_{i}=1.0 ; \varepsilon=$ $1.0 ; \alpha_{i}$ is varied according to equation (3).

Duty ratio: $\gamma=0.6$.

\section{B. Verification of the Creation of Protoplasmic Streaming}

In order to confirm the validity of the proposed method, simulations were performed under the above problem settings. Figure 5 (a) and (b) show representative results obtained under the condition where the number of modules was set to 92 and 563, respectively. The thick circles in the figures denote obstacles. These snapshots are in the order of the time transition (see from the left to right in each figure). As in the figures, the modular robot can successfully negotiate the environmental changes without losing the coherence. These results provide us the following three points that have to be noted. First, as we clearly see from the figure (b), the traveling wave stemming from the phase distribution created through the mutual entrainment gradually becomes conspicuous (see time step 1000 in the figure), and the right and left outer sections in the module group start moving toward the center. As a result, the protoplasmic streaming is emerged by causing the connection and disconnection among the modules. It should be noted that the dynamics of the connection mechanism provided by the functional materials is fully exploited in the process. Second, the way of negotiating the environment seems significantly different: the modular robot in the figure (a) passes through the obstacles by narrowing the width of the entire system, whilst the one in the figure (b) negotiates its environment by enclosing the obstacles. Note that these behavior are not pre-programmed, but are totally emergent. Third and finally, the effect of surface tension contributes to maintain the coherence of the entire system. Around the time step of 3000 in the figure (a), we temporarily turned off the goal light. As we see from the figure, the modular robot starts to form a circular shape. This is due to the effect of surface tension. We have observed that the

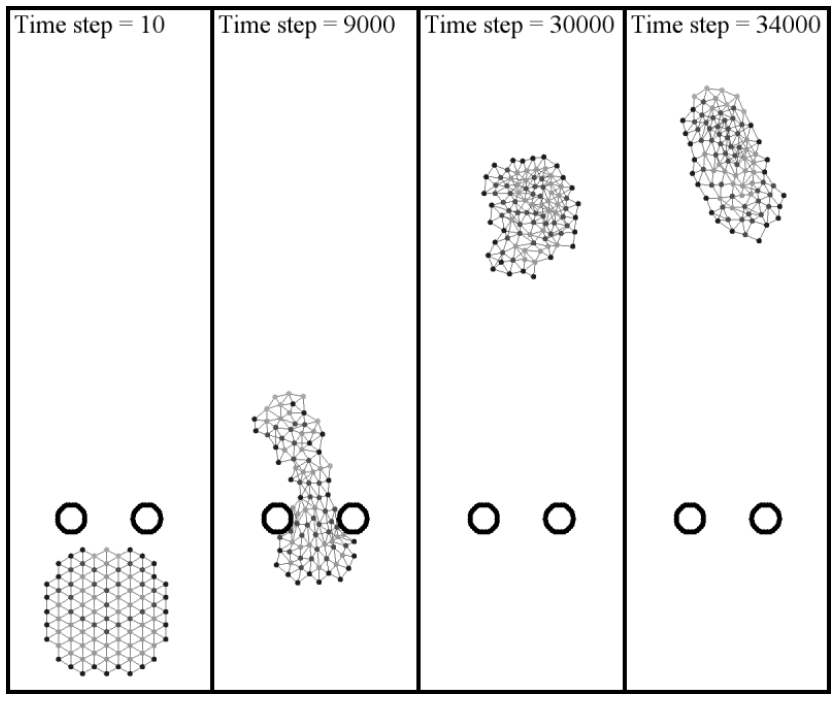

(a) The number of the modules: 92

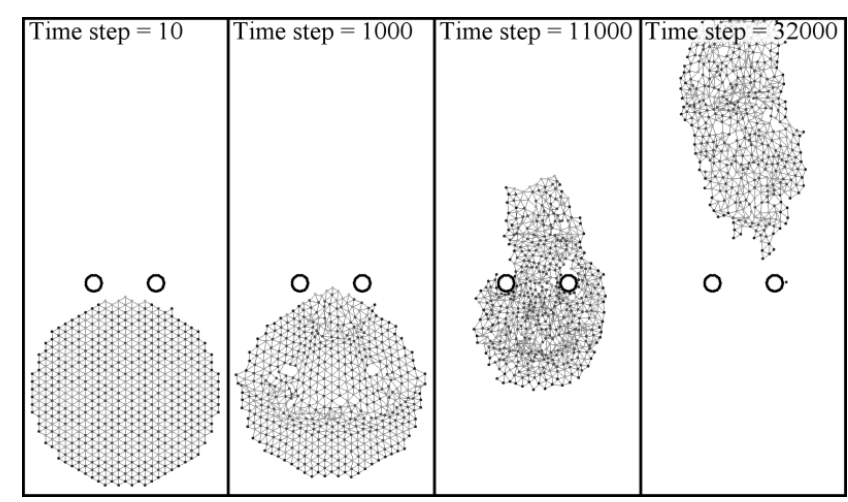

(b) The number of the modules: 563

Fig. 5. Representative data of the transition of the morphology (see from the left to right in each figure). The thick circles in the figures are the obstacles.

modular robot cannot maintain the coherence without this effect.

\section{CONCLUSION AND FURTHER WORK}

This paper discussed a decentralized control method enabling a modular robot to control its morphology in real time by explicitly exploiting an emergent phenomena stemming from the interplay between the control and mechanical dynamics. To this end, we focused on the functional material and the phase distribution created through the mutual entrainment among the VDP oscillators by utilizing the former as a mechanism for inter-module connection control and the latter as a core mechanism for locomotion pattern generation. Simulations conducted indicate that the proposed algorithm can induce a stable protoplasmic streaming inside the modular robot, which allows us to successfully control the morphology in real time according to the situation encountered without losing the coherence of the entire system. It should be noted that the control method discussed here does not control the connection/disconnection among the modules explicitly. 
This is totally an emergent phenomenon.

In order to control the morphology of a modular robot having a great degree of freedom in real time, the concept of exploiting the protoplasmic streaming inside the modular robot derived from the mutual entrainment among the VDP oscillators and the dynamic characteristics of the functional material introduced in this paper is considered to play an extremely important role in simplifying the necessary control algorithm. In addition, the protoplasmic streaming discussed here is emergent as the number of modules increases. This satisfies one of the important aspects of emergent phenomena: "a quantitative change leads to a qualitative change". To our knowledge, this is a first study explicitly based on this idea in the field of modular robots. To verify the feasibility of our proposed method, an experiment with a real physical modular robot is significantly important. This is currently under investigation.

\section{REFERENCES}

[1] T. Fukuda and Y. Kawauchi, Cellular robotic system (CEBOT) as one of the realization of self-organizing intelligent universal manipulators, Proc. of IEEE ICRA, pp.662-667, 1990.

[2] M. Yim, C. Eldershaw, Y. Zhang, and D. Duff, Self-Reconfigurable Robot Systems: PolyBot, Journal of the Robotics Society of Japan, Vol.21, No.8, pp.851-854, 2003.

[3] Hydra Project (EU Project): http://www.hydra-robot.com

[4] A. Castano, W.-M. Shen, and P. Will, CONRO: Towards Miniature Self-Sufficient Metamorphic Robots, Autonomous Robots, pp.309324, 2000.

[5] H. Kurokawa, E. Yoshida, A. Kamimura, K. Tomita, S. Yoshida, and H. Kokaji, Autonomous Modular Robot M-TRAN for Metamorphosis and Locomotion (in Japanese), Journal of the Robotics Society of Japan, Vol.21, No.8, pp.855-859, 2003.

[6] Y. Kakazu and N. Takahashi, Biomimetics and Modular Robotic System (in Japanese), Journal of the Robotics Society of Japan, Vol.21, No.8, pp.839-842, 2003. 